\title{
Article
}

\section{Coupling dye-integrated polymeric membranes with smartphone detection to classify bacteria}

Bueno, L., Cottell, A., Reddy, Subrayal M and Paixao, T.R.L.C.

Available at http://clok.uclan.ac.uk/14370/

Bueno, L., Cottell, A., Reddy, Subrayal M ORCID: 0000-0002-7362-184X and Paixao, T.R.L.C. (2015) Coupling dye-integrated polymeric membranes with smartphone detection to classify bacteria. RSC Advances, 5 (119). pp. $97962-$ 97965.

It is advisable to refer to the publisher's version if you intend to cite from the work. http://dx.doi.org/10.1039/c5ra19874g

For more information about UCLan's research in this area go to http://www.uclan.ac.uk/researchgroups/ and search for <name of research Group>.

For information about Research generally at UCLan please go to http://www.uclan.ac.uk/research/

All outputs in CLoK are protected by Intellectual Property Rights law, including Copyright law. Copyright, IPR and Moral Rights for the works on this site are retained by the individual authors and/or other copyright owners. Terms and conditions for use of this material are defined in the policies page. 


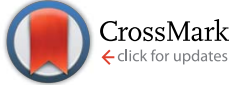

Cite this: RSC Adv., 2015, 5, 97962

Accepted 5th November 2015

DOI: $10.1039 / \mathrm{c} 5 \mathrm{ra} 19874 \mathrm{~g}$

www.rsc.org/advances

\section{Coupling dye-integrated polymeric membranes with smartphone detection to classify bacteria $\uparrow$}

\author{
Lígia Bueno, ${ }^{a}$ Alison Cottell, ${ }^{b}$ Subrayal M. Reddy ${ }^{c}$ and Thiago R. L. C. Paixão*a
}

We report the use of a colorimetric plastic-based device to discriminate four pathogenic bacteria: Klebsiella pneumoniae, Proteus vulgaris, Proteus mirabilis and Escherichia coli. The colour changes of the plastic membranes were analysed with RGB values extracted from dye-integrated polymeric membrane images obtained with a smartphone and used as input data for non-supervised pattern recognition methods.

The Enterobacteriaceae are a large family of bacteria commonly found in the intestines of mammals and some of the constituent species are associated with bacterial food spoilage and foodborne illness. ${ }^{1}$ Contamination of food with Enterobacteriaceae may occur because of inadequate hygiene or care during food preparation; contamination from the natural environment and, in rare cases, due to deliberate adulteration of foodstuffs. In this study, the four species of Enterobacteriaceae used are Klebsiella pneumonia (KP), Proteus vulgaris (PV), Proteus mirabilis and Escherichia coli. Proteus mirabilis has been reported in some food poisoning cases with pork balls. ${ }^{2}$ Klebsiella pneumonia can be destructive to human lungs through inflammation or haemorrhaging and it has been additionally identified with food poisoning cases. ${ }^{3}$ Escherichia coli is one of the most well-known bacteria and naturally found in vegetables and water and can also induce spoilage of some foods subsequently causing illness.,5

During their growth process, these bacteria produce volatile organic compounds (VOCs) ${ }^{6,7}$ that are unique to each genus or species. The VOCs produced can therefore be viewed as a chemical fingerprint for these organisms and can subsequently be used to help to detect the pathogens in, for example,

anstituto de Química, Universidade de São Paulo, São Paulo, SP, Brazil 05500-000. E-mail:trlcp@iq.usp.br

${ }^{b}$ Department of Microbiology and Cellular Sciences, FHMS, University of Surrey, Guildford, UK, GU2 $7 X H$

${ }^{c}$ Department of Chemistry, FEPS, University of Surrey, Guildford, UK, GU2 7XH

$\uparrow$ Electronic supplementary information (ESI) available. See DOI: 10.1039/c5ra19874g spiked blood culture. ${ }^{8}$ In the literature, there are different types of pattern array sensor, called optoelectronic nose or electronic noses, which uses this approach to transduce a chemical fingerprint in a readable output format to discriminate different samples. ${ }^{9-13}$ Currently, the majority of electronic nose devices ${ }^{9,14,15}$ that can discriminate gas compounds cannot be incorporated into or they are not compatible with the food packaging process. We propose a polymer-based thin dye film capable of responding to the in situ production of VOCs which can be easily integrated into food packaging. This communication reports the application of a colorimetric plastic-based device to classify four pathogenic bacteria: KP, PV, PM and EC using a smartphone as a detector. Based on our knowledge, this is the first time that an in situ simple procedure is used to discriminate these four microorganisms using a smartphone. This type of detector is receiving widespread attention by the scientific community ${ }^{16}$ due to its characteristics, of convenience, portability and low cost. Cellulose acetate polymer was dissolved in acetone $(0.1 \mathrm{~g}$ per $5 \mathrm{ml})$ in glass vials and the mixture shaken using a vortex until complete dissolution. In order to colour them, the dyes (methyl red, alizarin, bromophenol blue, thymol blue, chlorophenol red) were used individually at $1 \mathrm{mg}$ per $\mathrm{ml}$ of solution. After complete solubilization of the dye, $150 \mu \mathrm{l}$ of plasticizer Tween ${ }^{\circledR} 20$ was added and the solution further agitated. The resulting solution was poured into a glass Petri dish $(5 \mathrm{~cm}$ inner diameter), and the solution swirled to produce full surface coverage by solution. The Petri dish was then covered with its lid and placed on a flat surface and left under a fume hood at room temperature overnight until complete evaporation of the solvent and formation of the membrane structure. The membranes were cut with a cork-borer (11 mm diameter) and the cutting placed on a separate Petri dish, and stored in the close chamber to avoid exposition to other chemical compounds found in the laboratory. The detailed process was recently reported by us in the literature. ${ }^{17}$ To this end, we have prepared cellulose acetate membranes with five different $\mathrm{pH}$ indicators (alizarin, bromophenol blue, chlorophenol red, methyl red and thymol blue) 
immobilized within the membrane structure with Tween 20 surfactant as a plasticizer. The detailed process was recently reported by us in the literature. ${ }^{\mathbf{1 7}}$

Prior to the exposure on to the polymeric membranes the four bacterial species were sub-cultured overnight at $37{ }^{\circ} \mathrm{C}$ on nutrient agar. Cell suspensions of the bacteria were prepared in bacteriological saline and adjusted to a cell density of 3-12.5 $\times$ $10^{8}$ CFU (Colony-forming unit) using spectrophotometry, and confirmed with the Miles-Misra serial dilution technique. Aliquots of each of the cell suspensions was spread over the surface of $4.5 \mathrm{~mm}$ diameter Petri dishes containing the dyeintegrated polymeric membrane array in the lid and incubated at $25{ }^{\circ} \mathrm{C}$ and $37{ }^{\circ} \mathrm{C}$. Nutrient agar plates that were prepared and incubated identically, but did not contain bacteria, were used as controls.

The VOCs released can change the $\mathrm{pH}$ of an interfacing medium and, in this case, they can change the membrane's colour in a predictable way for each studied microorganism. Fig. 1 shows the distinct colour difference maps obtained for each evaluated bacterium, the technique allowing it to be possible to distinguish the bacteria even by the naked eye. To achieve the colour difference maps, it was necessary to make a subtraction of red, green and blue (RGB) values between membranes images after exposure to bacteria and RGB values from a blank (exposed membranes only containing a nutrient agar plate; no bacteria). RGB values were extracted using a home-made software (App) developed to extract RGB from coloured materials which was installed in an iPhone ${ }^{\circledR} 4 \mathrm{~S}$. More details of the software can be found here. ${ }^{17,18}$ Additionally, a closed chamber was used to control the light conditions during image acquisition and to fix the focal distance. The light was produced by four white LEDs passed through a series of transparent sandblasted poly(methyl methacrylate) sheets to optimize the distribution of light over the sample. The RGB values for each spot were collect from the centre of the spot $(260$ $\times 260$ pixels region) and the standard deviation for each RGB components were measured too. Each difference map is represented by 15 values (each difference map contains 5 dyes and each dye yields 3 values). The colour difference maps were used as input data for unsupervised chemometric tools, namely PCA (Principal Component Analysis) and HCA (Hierarchical Cluster Analysis), using Statistica software (Statsoft, USA) in a desktop. More information about the system can be obtained in the literature. ${ }^{17,18}$

The different colour patterns obtained in Fig. 1 are related to the different VOCs released by the a given bacterium during its growth and colonisation stages, and directly related to different amines released during these stages. ${ }^{6,7,17,19}$ Rosová and collaborators $^{6}$ have shown that bacterial volatile fatty acid patterns could be useful to discriminate bacteria. In their work, they demonstrate that PM exudes acetic acid; EC produces acetic acid and propionic acid and KP releases acetic acid, propionic acid and isovaleric acid. Volatile amines can also be detected as proved in our previous work. ${ }^{\mathbf{1 7}}$ Therefore, a mixture of volatiles or even a single molecule exudate compound will result in a colour change of the plastic device resulting in a unique pattern for bacterial recognition. In order to make the
A

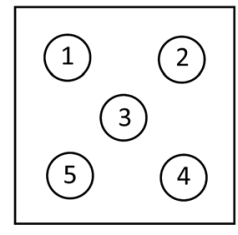

1) Alizarin

2) Bromophenol Blue

3) Chlorophenol Red

4) Methyl Blue

5) Thymol Red

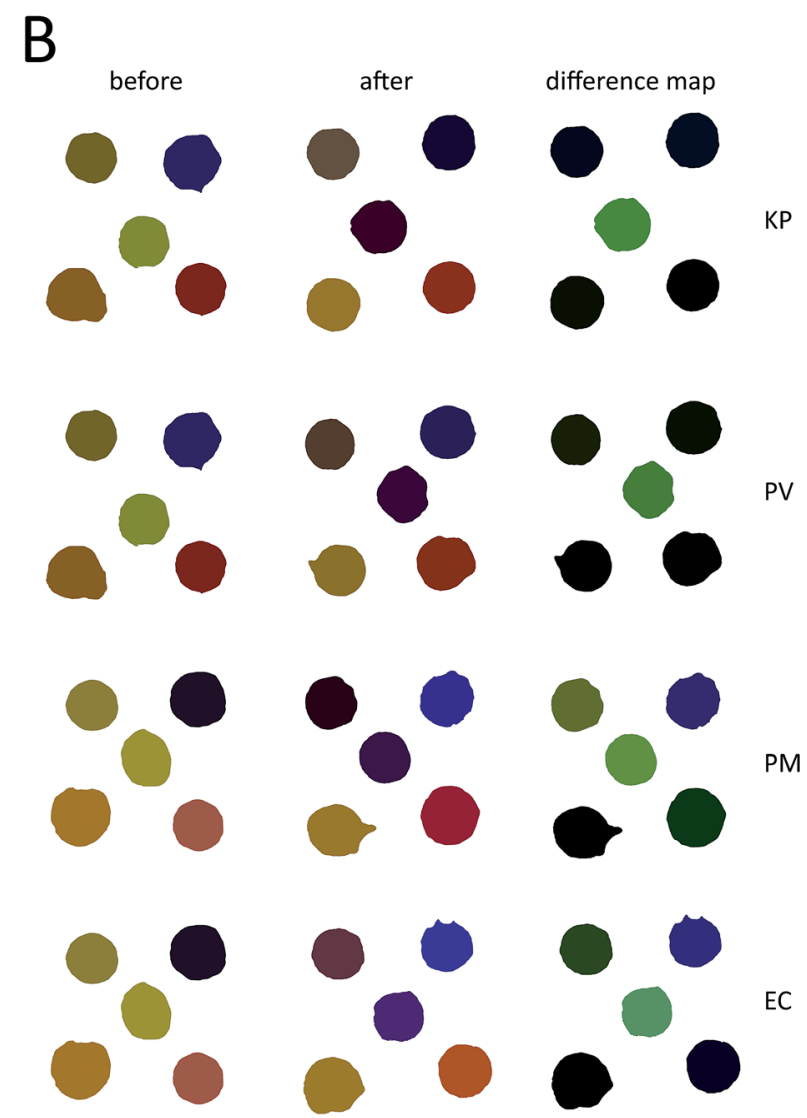

Fig. 1 Schematic representation of the position of each $\mathrm{pH}$ indicator on the device and the colour changes profile of the sensor array (A). In (B), we show the images before and after exposure of the plastic device to Klebsiella pneumoniae (KP), Proteus vulgaris (PV), Proteus mirabilis (PM) and Escherichia coli (EC), as well as the difference map obtained by the subtraction of the 'after exposure' RGB values from the 'before exposure' RGB values.

discrimination noticeable in our work, RGB values from the colour difference maps were used as input data to chemometric tools, Principal component analysis (PCA) and Hierarchical component analysis (HCA). Fig. 2 presents a clear discrimination among all four studied bacteria using only the first two principal components, with more than $90 \%$ of the total information collected from RGB values extracted. Such discrimination was quantitatively confirmed by using a HCA made with the same data used to obtain the score plot reported in Fig. 1 (Fig. S1 ESI $\dagger$ ), where each strain is located in a different group and there are no misclassifications.

A loading plot (Fig. S2 ESI $\dagger$ ) was made in order to understand which variables are responsible for the discrimination obtained 


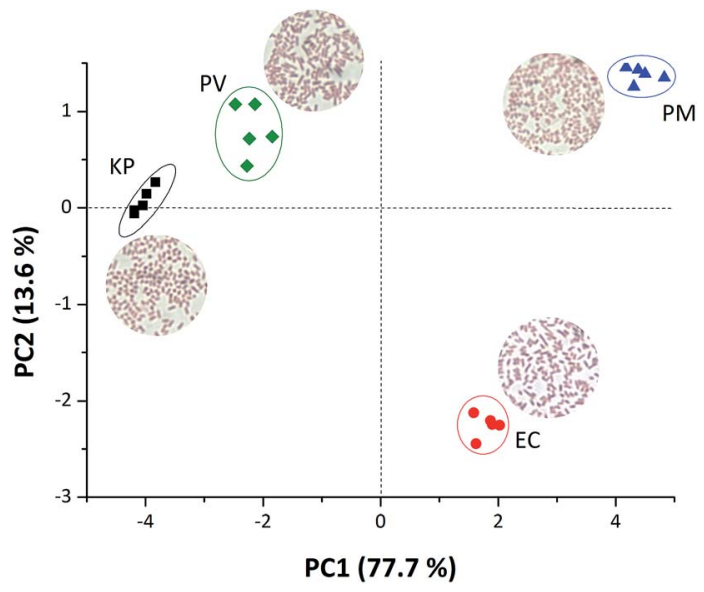

Fig. 2 PCA score plot obtained from the RGB values extracted from the coloured membranes with $5 \mathrm{pH}$ indicators in contact with four different bacteria species: Klebsiella pneumoniae (KP), Proteus vulgaris (PV), Proteus mirabilis (PM) and Escherichia coli (EC) incubated at 37 ${ }^{\circ} \mathrm{C}$. Insets show the optical image of the bacteria strains.

in Fig. 1. Fig. $\mathrm{S} 2 \uparrow$ shows that the blue component in chlorophenol red and bromophenol blue, as well as, the green component in bromophenol blue are the most important variables to keep EC species in the second quadrant. Previously, ${ }^{17}$ we showed that the blue component of chlorophenol red is important to discriminate isopentylamin $\mathrm{e}^{19}$ the latter VOC being indicative of EC colonization at $37^{\circ} \mathrm{C}$. The dyes alizarin, thymol red and bromophenol blue are responsible for the KP and PV discrimination. It was previously shown that the release of isobutylamine could be detected by thymol red and methyl blue dyes which is in agreement with the literature for the anaerobic decarboxylation of $\mathrm{L}$-valine by PV producing isobutylamine. ${ }^{19}$ Notwithstanding, the discrimination of PM, the colour parameters extracted from the bromophenol blue and chlorophenol red dyes are important, concluding that all variables used are important for the discrimination process of the microorganisms studied here.

With the idea to develop food packaging-integrated smart indicators to detect food spoilage at room temperature, experiments were conducted at $25^{\circ} \mathrm{C}$ to simulate the bacterial growth and to evaluate the possibility to discriminate microorganisms under ambient conditions. Fig. 3 shows good discrimination amongst all considered bacteria, with each one being placed in a different quadrant, even when using about $60 \%$ of the total information. Although this temperature is not the best condition for bacterial growth, the results prove that the proposed device is able to discriminate microorganisms. As with the $37^{\circ} \mathrm{C}$ experiments, HCA graph (Fig. S3 ESI $\dagger$ ) was plotted to confirm the PCA plot in the $25^{\circ} \mathrm{C}$ tests and in this case we do not have any misclassification, and correctly identifying each strain group evaluated.

A supervised classification using $k$-NN was performed to test the ability of the proposed method to classify the four bacteria discriminated in this study. The $k$-NN algorithm classifies patterns based only on the calculated distance between points

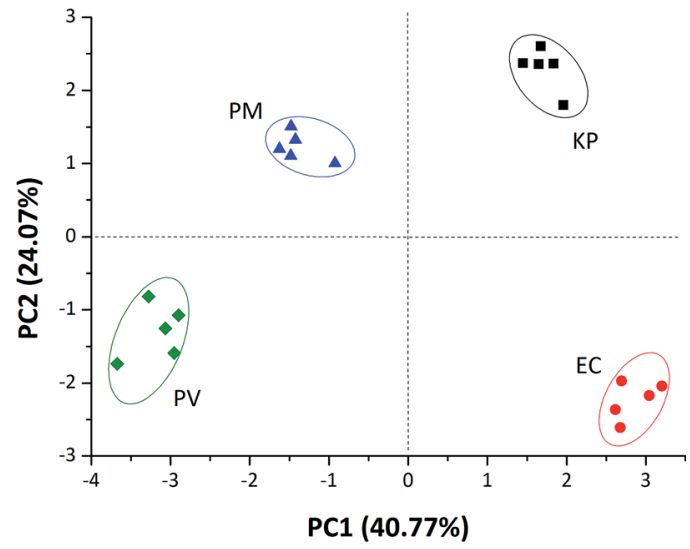

Fig. 3 PCA score plot obtained from the RGB values extracted from the coloured membranes with $5 \mathrm{pH}$ indicators in contact with four different bacteria species: Klebsiella pneumoniae (KP), Proteus vulgaris (PV), Proteus mirabilis (PM) and Escherichia coli (EC) incubated at $25^{\circ} \mathrm{C}$.

using the n-dimensional pattern space. In this method, an unknown sample is classified according to the majority vote of its $k$ nearest neighbours in the training set. Therefore, the $k$-NN model was trained using the data from 16 samples (4 from each single bacterium). First, the choice of $k$ nearest neighbours to be used was evaluated using a leave-one out cross-validation method. In this approach, the model is repeatedly refit leaving one of the 16 samples out as a single observation and then used to derive a prediction for the left-out observation using the rest of the samples (15) as a training set. The accuracy of the predictions made by left-out observations is estimated for each possible value of $k$. Fig. 4 shows the cross-validation accuracy of the $k$-NN model for different $k$ values in the training set. As can be seen, the best cross-validation accuracy was achieved when $k=1$.

To verify the accuracy of the proposed model and the best value of $k$, four new samples of each bacteria studied here were prepared and the predictive power of the model was tested. Table 1 reports a confusion matrix, the true class value against

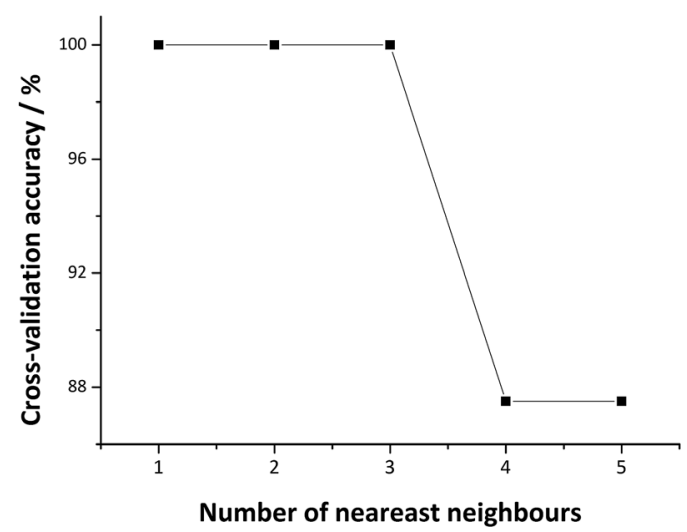

Fig. 4 Cross-validation accuracy plot showing the number of $k$ nearest neighbour to yield the highest percent of accuracy. 
Table 1 Confusion matrix for the $k-N N$ classification results for the bacteria samples

\begin{tabular}{lllll}
\hline & \multicolumn{2}{l}{ Prediction } & & \\
\cline { 2 - 5 } Samples & KP & EC & PM & PV \\
\hline KP & $100 \%$ & & & \\
EC & & $100 \%$ & & \\
PM & & & $100 \%$ & $100 \%$ \\
PV & & & &
\end{tabular}

the class value predicted by the $k$-NN model using RGB values of the five membranes as input. As shown in Table 1, for all four analysed samples, the percentage of correct prediction of the unknown samples was $100 \%$, indicating an excellent accuracy of the proposed method to classify bacteria.

A colorimetric device was successfully applied to discriminate and classify four bacteria strains after incubation at $37{ }^{\circ} \mathrm{C}$ and $25{ }^{\circ} \mathrm{C}$ using a combination of polymer membrane, five dyes and smartphone technology. The data were analysed with chemometric tools (PCA, HCA and $k$-NN) and the results demonstrated the potential to apply this technique in food packaging control to detect bacteria.

\section{Acknowledgements}

The authors are grateful to the funding agencies CAPES (Grant number: 3359/2014 PRÓ-FORENSES Edital 25/2014), FAPESP (Grant number: 2011/23355-3 and 2014/50792-3), Office of Naval Research (Award number N62909-15-1-N045), Royal Society, UK (Grant number: EI130745), and CNPq (Grant number: 444498/ 2014-1) for supporting this research.

\section{Notes and references}

1 M. E. Nyenje, C. E. Odjadjare, N. F. Tanih, E. Green and R. N. Ndip, Int. J. Environ. Res. Public Health, 2012, 9, 26082619.
2 Y. Wang, S. Zhang, J. Yu, H. Zhang, Z. Yuan, Y. Sun, L. Zhang, Y. Zhu and H. Song, Food Control, 2010, 21, 302-305.

3 J. M. Sabota, W. L. Hoppes, J. R. Ziegler, H. DuPont, J. Mathewson and G. W. Rutecki, Am. J. Gastroenterol., 1998, 93, 118-119.

4 N. J. C. Strachan, G. M. Dunn, M. E. Locking, T. M. S. Reid and I. D. Ogden, Int. J. Food Microbiol., 2006, 112, 129-137.

5 P. Ferrier and J. C. Buzby, Food Control, 2014, 38, 227-234.

6 J. Julak, E. Prochazkova-Francisci, E. Stranska and V. Rosová, J. Microbiol. Methods, 2003, 52, 115-122.

7 E. Tait, J. D. Perry, S. P. Stanforth and J. R. Dean, J. Chromatogr. Sci., 2014, 52, 363-373.

8 S. H. Lim, S. Mix, Z. Xu, B. Taba, I. Budvytiene, A. N. Berliner, N. Queralto, Y. S. Churi, R. S. Huang, M. Eiden, R. A. Martino, P. Rhodes and N. Banaei, J. Clin. Microbiol., 2014, 52, 592-598.

9 Q. Chen, H. Li, Q. Ouyang and J. Zhao, Sens. Actuators, B, 2014, 205, 1-8.

10 J. R. Askim and K. S. Suslick, Anal. Chem., 2015, 87, 78107816.

11 W. Yang, P. Wan, M. Jia, J. Hu, Y. Guan and L. Feng, Biosens. Bioelectron., 2015, 64, 547-553.

12 J. R. Askim, M. Mahmoudi and K. S. Suslick, Chem. Soc. Rev., 2013, 42, 8649-8682.

13 Y. Zhang, J. R. Askim, W. Zhong, P. Orlean and K. S. Suslick, Analyst, 2014, 139, 1922-1928.

14 J. W. Grate, Chem. Rev., 2000, 100, 2627-2647.

15 F. Roeck, N. Barsan and U. Weimar, Chem. Rev., 2008, 108, 705-725.

16 X. Wang, Y. Qin and M. E. Meyerhoff, Chem. Comm., 2015, 15176-15179.

17 L. Bueno, G. N. Meloni, S. M. Reddy and T. R. L. C. Paixão, RSC Adv., 2015, 5, 20148-20154.

18 M. O. Salles, G. N. Meloni, W. R. de Araujo and T. R. L. C. Paixão, Anal. Methods, 2014, 6, 2047-2052.

19 N. A. M. Eskin, Biochemistry of Foods, Elsevier Science, 1990. 